\title{
Hepatectomy and liver regeneration: from experimental research to clinical application
}

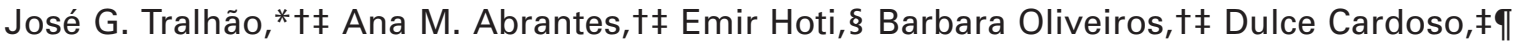 \\ François Faitot, ${ }^{*}$ César Carvalho, ${ }^{*}$ Maria F. Botelho†‡ and Francisco Castro-Sousa* $¥$ \\ *Department of Surgery A, Coimbra University Hospital, Faculty of Medicine, University of Coimbra, Coimbra, Portugal \\ †Biophysics Unit, IBILI, Faculty of Medicine, University of Coimbra, Portugal \\ $\ddagger$ Centre of Investigation on Environment, Genetics and Oncobiology (CIMAGO), Faculty of Medicine, Coimbra, Portugal \\ §Liver Transplant Unit, Saint Vincents' University Hospital, Dublin, Ireland \\ INuclear Medicine Department, Coimbra University Hospital, Coimbra, Portugal and \\ **Unité INSERM U785, Hopital Paul Brousse, Villejuif, France
}

\section{Key words}

99mTc-mebrofenin, bromodeoxyuridine

deoxyribonucleic acid (BrdU), hepatectomy, hepatic

extraction fraction, human liver regeneration kinetics, oncological therapeutic approaches.

\section{Correspondence}

José G. Tralhão, Serviço de Cirurgia A, Centro

Hospitalar e Universitário de Coimbra, Faculdade de

Medicina, Universidade de Coimbra, Praceta Mota

Pinto, 3000-075 Coimbra, Portugal.

Email: jglrt@hotmail.com

J. G. Tralhão MD, PhD; A. M. Abrantes MSc; E. Hoti $\mathrm{MD}$; B. Oliveiros MSc; D. Cardoso BSc; F. Faitot MD;

C. Carvalho MD; M. F. Botelho MD, PhD;

F. Castro-Sousa MD, PhD, FACS.

Accepted for publication 24 March 2013

doi: 10.1111/ans. 12201

\begin{abstract}
Background: The mechanisms and kinetics of hepatic growth have continuously been investigated. This study concerns liver regeneration in animal and patients who underwent partial hepatectomy evaluated by the hepatic extraction fraction (HEF) calculated through radioisotopic methods.

Methods: Thirty normal Wistar rats were submitted to an $85 \%$ hepatectomy, and 95 patients with primary and secondary liver tumours were included. In animal study, the liver regeneration kinetics was assessed by HEF using $99 \mathrm{mTc}-$ mebrofenin, the ratio liver/bodyweight and by using bromodeoxyuridine deoxyribonucleic acid incorporation. In patient study, the liver regeneration was evaluated by calculation of HEF before surgery, 5 and 30 days after hepatectomy.

Results: In animal, we verified a positive correlation between HEF kinetics and liver/bodyweight ratio or hepatocyte proliferation evaluated by bromodeoxyuridine deoxyribonucleic acid staining after $85 \%$ hepatectomy. In the clinical arm, no statistical differences of the HEF before hepatectomy, 5 and 30 days after hepatectomy, were observed.

Conclusions: Our results support the view that human liver regeneration commences early, is fast, non-anatomical and functionally complete 5 days after hepatectomy. The fast functional liver regeneration may have a high clinical impact particularly concerning the post-operative oncological therapeutic approaches.
\end{abstract}

\section{Introduction}

Liver regeneration is defined as an orchestrated response induced by specific external stimuli, leading to sequential changes in gene expression, growth factors production, as well as morphological structure (anatomy). ${ }^{1-3}$ Liver regeneration occurs through the proliferation of all existing mature cellular populations composing the intact organ. These include hepatocytes, biliary epithelial cells, fenestrated endothelial cells with large cytoplasmic gaps that allow maximal contact between circulating blood and hepatocytes, Kupffer cells, oval cells, liver precursor cells, and Ito cells. These cells synthesize connective tissue proteins and secrete several growth factors, such as hepatocyte growth factor (HGF), epidermal growth factor receptor ligands, epidermal growth factor, transforming growth factor, alpha tumour necrosis factor and acidic fibroblast growth factor. Besides this cellular intervention, humoral involving interleukin 6, norepinephrine, insulin and bile acids are also important..$^{4-8}$

The mechanisms and kinetics of hepatic growth have continuously been investigated by using either primary hepatocyte cell cultures or partially hepatectomized animals, or by evaluating liver volumes in recipient or donor patients after living donor transplantation, as well as in patients following liver resections. ${ }^{9-13}$

As we know, liver resection remains the treatment of choice in a number of primary and secondary liver tumours, and hepatectomy is currently considered the standard of care and remains the only 
potential curative therapy for liver metastases of colorectal cancer. Complete surgical resection of isolated or multiple hepatic metastases has been associated with a 26-51\% 5-year survival, which confers a clear survival advantage over $0-5 \%$ survival in patients who do not undergo liver resection. ${ }^{1-3}$ Similarly, liver resection can be considered the principal therapy for a specific group of patients with hepatocellular carcinoma. Although liver transplantation is accepted as the best curative option for carefully selected patients with hepatocellular carcinoma, this treatment cannot always be offered due to either patients' advanced age or scarcity of liver donors, and as a result, even in this group, hepatic resection continues to be the treatment of choice for the majority of patients (yielding 5-year survival rates as high as $60 \%$ in some series). ${ }^{4-6}$

Currently, nuclear medicine methodologies are able to give dynamic functional information, with the possibility of lesion characterization and also cell function. In this context, the $99 \mathrm{mTc}$ mebrofenin, a derivative of iminodiacetic acid labelled with $99 \mathrm{mTc}$, is transported into the hepatocyte by a high-capacity carriermediated anionic clearance mechanism, organic anion transporter polypeptide. After the uptake, acid imunodiacetic derivative is transported into the bile canaliculi by active membrane transport. These compounds will have the same hepatocyte uptake, transport and excretion pathways as bilirubin. Using $99 \mathrm{mTc}$-mebrofenin, a derivative of imunodiacetic acid labelled with $99 \mathrm{mTc}$ is possible to evaluate the hepatic extraction fraction (HEF) after intravenous injection. The presence of radiotracer in the excreted biliary fluid represents good hepatocyte function, that is tracer extraction and excretion. ${ }^{14-16}$ Changes in hepatocyte function modify the tracer cellular management, reflecting the dynamic process referred to. Therefore, nuclear imaging, which also provides information on biliary kinetic, is a good investigating alternative for the hepatocellular function.

Based on this, we conducted a combined experimental and clinical study to evaluate the liver regeneration using quantification of HEF after partial hepatectomy.

\section{Methods}

\section{Animal study}

Thirty male Wistar rats of 2 months of age were used for the experimental research. The animals were fed a laboratory diet with water and food ad libitum until surgery. They were kept under constant environment conditions with 12-h light-dark cycle. All the procedures were performed in accordance with the national and international animal care laws, and were approved by the local animal ethics committee.

The induction of liver regeneration by partial hepatectomy depends on the reduction of functional liver mass. In the rats, the liver mass makes up circa $4 \%$ of the bodyweight, the individual liver lobes constant proportions and each having a long vascular hilum with segmental portal vein, hepatic artery and bile duct. ${ }^{13}$ The animal model adopted was a major hepatectomy (partial hepatectomy of $85 \%$ ), which was already described by Cantré et al., referred this hepatectomy as an excellent model of liver regeneration, like those who reported $70 \%$ hepatectomy in rat models.,

All the animals were anaesthetized with methoxyflurane and submitted to the major hepatectomy. Briefly, the abdomen was entered through a midline incision, and the liver was freed from its ligaments. The left lateral lobe, median lobe and right superior lobe were then resected by placing 3/0 suture ties most proximally to their origin (left lateral and median lobes), or at short distance from their origin (right superior lobe), guaranteeing adequate blood flow to neighbouring residual lobe. ${ }^{9}$ Care was taken to avoid disruption of the hilar structures, including portal vein, hepatic artery and biliary tract. No animal was excluded due to mortality or morbidity. After surgery, the animals were divided into five subgroups according to the time they were sacrificed post hepatectomy: $12 \mathrm{~h}$ (six animals), 1 day (six animals), 2 days (six animals), 3 days (seven animals) and 5 days (five animals).

\section{Liver regeneration study in animal}

After sacrificing them, the remnant livers were procured and used to evaluate liver regeneration kinetics through DNA incorporation of 5-bromo-2'-deoxyuridine (BrdU), and the ratio of liver and bodyweight. In order to evaluate HEF, the animals were studied 5 days before surgery and $1 \mathrm{~h}$ before the sacrifice through an injection of $99 \mathrm{mTc}$-mebrofenin in a tail vein.

Overall liver regeneration was evaluated only on the animal models. This information was obtained by two different approaches: first, by weighting the animals and the remaining livers after the hepatectomy and the calculation of liver to bodyweight ratio (LW/ AW) after euthanasia; and second, by analysing the DNA synthesis using BrdU incorporation as described. ${ }^{12}$ Briefly, BrdU (Roche) was dissolved in phosphate-buffered saline and injected ( $2 \mathrm{~h}$ before sacrificing the animals) in a concentration of $0.1 \mathrm{mg} / \mathrm{g}$ of the animal bodyweight. Samples were collected and fixed in $10 \%$ tamponated formaldehyde in order to be evaluated by fluorescence microscopy to count the number of visualized nucleoli. For this analysis, five independent optical fields were studied.

\section{Patient study}

Ninety-five patients were enrolled in this study from January 2007 to May 2009. The mean age was $63.2 \pm 11.3$ years (range: $32-83$ ); 63 were men and 32 women. Sixty-nine patients had colorectal liver metastases, sixteen had hepatocellular carcinoma, two had cholangiocarcinoma, two had liver metastases from gastric cancer, two had hepatic hemangioma and four patients had other tumours. Ten patients were cirrhotic, diagnosed by radiological and histological investigations.

The selection of patients for surgery was based on liver functional status, radiological evidence of tumour resectability using computer tomography (CT) or magnetic resonance, study of hepatic volumetry by $\mathrm{CT}$, absence of major contraindications precluding surgery, and in the cases of hepatocellular carcinoma patients had to be outside the Milan criteria ${ }^{17}$ and classified as Child-Pugh score grade A. ${ }^{18}$

Thirty-five patients (37\%) underwent major hepatic resection (resection of three or more liver segments) (MAHR): right extended hepatectomy $(n=9)$, right hepatectomy $(n=11)$, central hepatectomy $(n=2)$, extended left hepatectomy $(n=1)$, left hepatectomy $(n=7)$ and trisegmentectomy $(n=5)$.

The remaining 60 patients $(63 \%)$ underwent minor hepatic resection $(M I H R)$ : bisegmentectomy $(n=22)$, left lobectomy $(n=8)$, segmentectomy $(n=25)$ and subsegmentectomy $(n=5)$. 
There was no immediate or 3-month post-operative mortality. Post-operative morbidity was $5.2 \%(n=5)$, which included wound infection in two patients, respiratory complications in two patients and bleeding peptic ulcer in one patient (treated conservatively).

The preoperative evaluation was done by standard liver biochemistry (plasma levels of prothrombin, albumin, alanine aminotransferase, aspartate aminotransferase, alkaline phosphatase, gamma-glutamyl transpeptidase, total and direct/indirect bilirubin, and lactic acid dehydrogenase) and Child-Pugh score.

All patients had HEF evaluated by radioisotopic methods with intravenous injection at antecubital vein of $99 \mathrm{mTc}$-mebrofenin, after fasting for at least $6 \mathrm{~h}$, before surgery (T0), 5 days after surgery (T5) and 1 month after surgery (T30). The HEF was evaluated 5 days after surgery because of convenience to patient.

Data analysis was performed considering major and minor hepatectomy patients globally and separately.

The study protocol was approved by the ethics committee of Coimbra University Hospital and Coimbra Faculty of Medicine. All patients were informed about the study and signed an informed consent.

\section{Calculation of HEF}

For HEF calculation, an iminodiacetic acid derivative, the 3-bromo2,4,6-trimethylphenylcarbamoilmethyl 1-iminodiacetic acid labelled with technetium-99 m (99mTc-mebofrenin) was used. The labelling procedure was performed by adding $1110 \mathrm{MBq}$ of $99 \mathrm{mTc}$ freshly eluted to a mebrofenin kit (GE-Amersham Health, Eindhoven, The Netherlands). For the patients studied, $185 \mathrm{MBq}$ was administrated, and for the animals studies the administered dose was $37 \mathrm{MBq}$. Related to dosimetry, the target organ that has the highest estimated absorbed dose is the large bowel (9.2 E-02 mGy/MBq), according to the Medical Internal Radiation Dose Committee guidelines. ${ }^{15,19}$

Data acquisition for experimental and clinical groups was performed by a single detector gamma camera (GE Maxicamera 400 AC, Milwaukee, WI, USA) coupled with a low-energy, highresolution, parallel-hole collimator, controlled by a genieACQ computer (GE, Milwaukee, WI, USA). Data were dynamically collected after the bolus injection of 99 mTc-mebrofenin for $128 \times 128 \times 16$ matrix, every second during the first minute, followed by 59 frames of $60 \mathrm{~s}$ each without zoom. After the dynamic acquisition, two 256 $\times 256$ static images, anterior and posterior, were performed with an individual duration of $67 \mathrm{~s}$ for decay correction.

All patients were positioned supine with the upper abdomen and cardiac area included in the gamma camera field of view. For the animals, the position was ventral decubitus directly over the collimator, with the whole body in the gamma camera field of view.

Energy discrimination was centred on $140 \mathrm{keV}$ with a $20 \%$ energy window. After acquisition, the data were transferred to a work station, eNTEGRA, for processing.

On the dynamic sequence of images, regions of interest (ROIs) were flagged over the cardiac region and right hepatic lobe, avoiding the gall bladder and main intrahepatic biliary ducts of patients. For the animals studied, the entire liver remnant is included as they do not have gall bladder. Time-activity curves were generated from these ROIs, and the HEF was calculated through deconvolution analysis of the first pass curve. This measurement also gives values of the T1/2 (time needed to reduced liver activity to half, assuming that the activity was instantaneously injected in the hepatic artery or portal vein as a Dirac delta function) and Tmax (time of maximum liver activity).

In the experimental arm involving animal subjects, normal HEF, the T1/2 and Tmax values were of $98 \pm 5 \%, 20.9 \pm 14.6 \mathrm{~min}$ and 18 $\pm 4.6 \mathrm{~min}$, respectively. Normal HEF values were of $99 \pm 0.4 \%$, the $\mathrm{T} 1 / 2$ values were $39 \pm 19 \mathrm{~min}$ and Tmax values were $15 \pm 6 \mathrm{~min}$ for the patients. ${ }^{15}$

This technical approach was used in both, experimental and clinical studies, to evaluate liver regeneration after hepatectomy, as described before in animal studies. ${ }^{19-21}$

\section{Statistical analysis}

Continuous data were presented as mean and standard deviation. Repeated measures analyses of variance were obtained by generalized linear models within effects (time) and one factor (hepatectomy) on variables HEF (\%), Tmax (min) and T1/2 (min) in all patients. $P$ values of $\leq 0.05$ were considered statistically significant.

For the correlation studies between HEF and BrdU incorporation or LW/AW ratio after the partial hepatectomy, Pearson correlation was applied and evaluated at 5\% significant level.

\section{Results}

\section{Animal studies}

\section{Correlation between post-operative HEF, T1/2, Tmax and liver/bodyweight ratio}

We verified a positive correlation between HEF kinetics and LW/AW ratio after the hepatectomy, and a negative correlation between T1/2 or Tmax and LW/AW ratio after hepatectomy (Fig. 1a-c).

\section{Correlation between post-operative HEF, T1/2, Tmax and BrdU incorporation}

A positive correlation between hepatocyte proliferation evaluated by BrdU staining and HEF after hepatectomy was obtained. Similarly, we observed a negative correlation between hepatocyte proliferation and $\mathrm{T} 1 / 2$ or Tmax after surgery (Fig. 2a-d).

\section{Patient studies}

Correlation between pre- and post-operative HEF, T1/2 and Tmax

Overall patient population. The mean HEF values in our series were $97 \pm 10 \%$ for T0, $98 \pm 8 \%$ for T5 and $99 \pm 4 \%$ for T30 $(P=$ $0.694)$. The mean Tmax values were $16 \pm 8 \mathrm{~min}$ for T0, $14 \pm 6 \mathrm{~min}$ for T5 and $14 \pm 5 \mathrm{~min}$ for T30 $(P=0.073)$. The mean T1/2 values were $36 \pm 22 \mathrm{~min}$ for $\mathrm{T} 0,86 \pm 105 \mathrm{~min}$ for $\mathrm{T} 5$ and $40 \pm 19 \mathrm{~min}$ for T30 $(P=0.0001)$.

Subgroup of patients treated by major resection. For the 35 patients who underwent MAHR, the HEF values were $97 \pm 5 \%$ (T0), $96 \pm$ $13 \%$ (T5) and $99 \pm 2 \%$ (T30) (0.472); the mean Tmax values were $17 \pm 7 \mathrm{~min}$ for $\mathrm{T} 0,14 \pm 6 \mathrm{~min}$ for $\mathrm{T} 5$ and $15 \pm 5 \mathrm{~min}$ for T30 (0.073); and the mean T1/2 values were $27 \pm 26 \min$ for $\mathrm{T} 0,89 \pm$ 
(a)

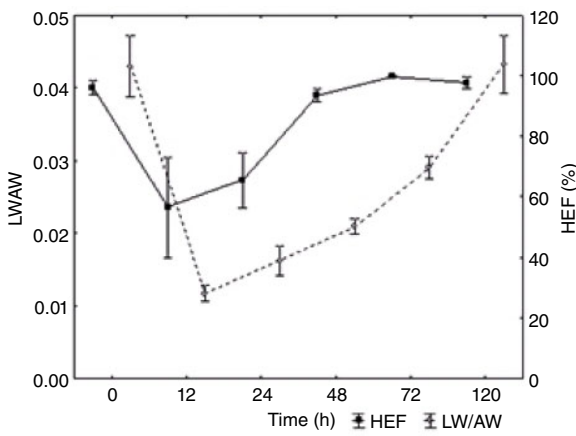

(c)

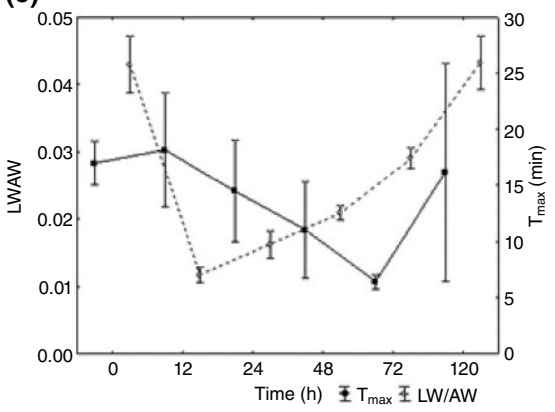

(b)

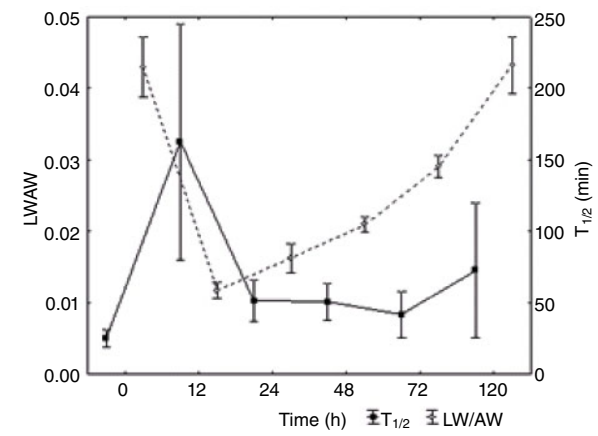

(d)

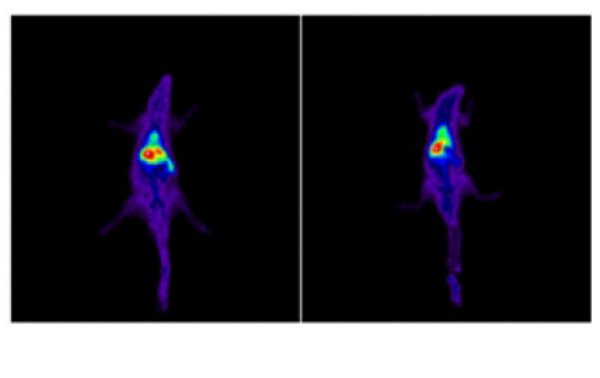

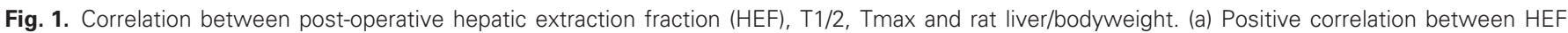

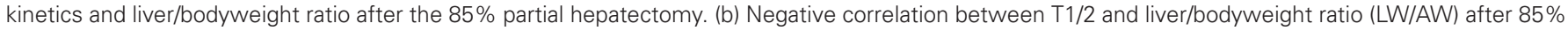

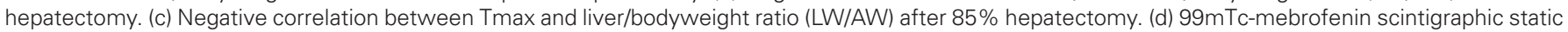
image in animal models before and $24 \mathrm{~h}$ after $85 \%$ partial hepatectomy.

$74 \mathrm{~min}$ for $\mathrm{T} 5$ and $42 \pm 9 \mathrm{~min}$ for $\mathrm{T} 30(P=0.001)$. There are significant differences only for the values of T1/2 in T5 when compared with T0 $(P=0.035)$ (Fig. 3a,b).

Subgroup of patients treated by minor resections. For the 60 patients who underwent $M I \mathrm{HR}$, the $\mathrm{HEF}$ values were $97 \pm 11 \%$ (T0), $99 \pm 2 \%$ (T5) and $99 \pm 5 \%$ (T30) $(P=0.879)$; the mean Tmax values were $15 \pm 9 \mathrm{~min}$ for $\mathrm{T} 0,14 \pm 6 \mathrm{~min}$ for $\mathrm{T} 5$ and $14 \pm 6 \mathrm{~min}$ for T30 $(P=0.098)$; and the mean T1/2 values were $39 \pm 19 \mathrm{~min}$ for $\mathrm{T} 0,85 \pm 122 \mathrm{~min}$ for $\mathrm{T} 5$ and $39 \pm 22 \mathrm{~min}$ for $\mathrm{T} 30(P=0.001)$. There are significant differences only for the values of T1/2 in T5 when compared with T0 $(P=0.04)$ (Fig. 3a,c).

\section{Discussion}

During the last two decades, there have been major advances in the understanding of the mechanisms of liver regeneration. This knowledge, obtained from laboratory research using animals, alongside with the use of data from solid organ transplantation in human, has been applied in different experimental studies as well as certain clinical situations. ${ }^{3-6}$ The research conducted so far has resulted in the identification of a number of factors, such as HGF, transforming growth factor- $\alpha$, and heparin-binding epidermal growth factorlike. ${ }^{1,3,4,6}$ Indeed, these factors play a very important role in enhancing and accelerating liver regeneration after liver resection or transplantation, and could positively influence livers affected by small-for-size syndrome or in injured livers secondary to trauma.
The induction of liver regeneration by partial hepatectomy depends on the reduction of functional liver mass. The model of $2 / 3$ hepatectomy in the rat devised many years ago by Higgins and Anderson has found broad acceptance. ${ }^{13}$ Therefore, the increment of the portion of liver resected increases the stimulus to liver regeneration, so this is the reason why a hepatectomy of $85 \%$ was used. Increasing the proportion of liver volume resected would increase the stimulus to liver regeneration, and this was the rationale why a hepatectomy involving up to $85 \%$ of liver parenchyma was performed in our experimental arm. On the other hand, $85 \%$ hepatectomy may mimic the changes of hepatic perfusion (e.g. portal hyperperfusion) associated with reduced-size livers that can be a source of complications as the small-for-size syndrome. ${ }^{9}$ This is characterized by severe liver dysfunction or even liver failure in the first post-operative week, and is consequently associated with high mortality rate. ${ }^{9}$

Our experimental results show that immediately after hepatectomy in the rat, the liver function decreases, with the lowest values being at $24 \mathrm{~h}$. After this time, the liver function recovers and returns to normal. These results suggest a correlation between liver function evaluated by $99 \mathrm{mTc}-$ mebrofenin and liver regeneration evaluated with BrdU staining and LW/AW ratio quantification. Our results are in line with the results published by others where no significant differences were found between $99 \mathrm{mTc}-$ mebrofenin uptake and conventional liver volume on days 3, 5 and 7 after $70 \%$ hepatectomy was performed in same animal model. ${ }^{19,21}$ Also, in this study, the authors demonstrated a good correlation between the 
(a)

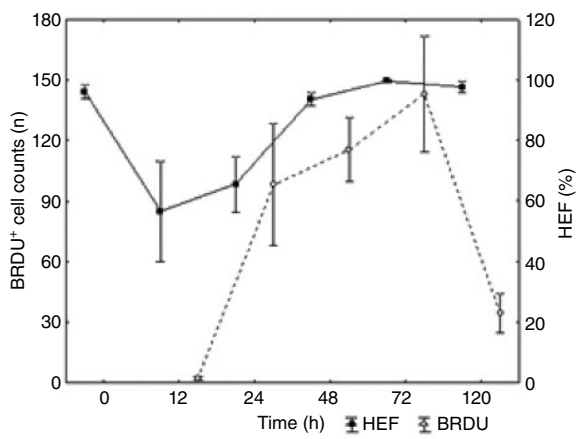

(c)

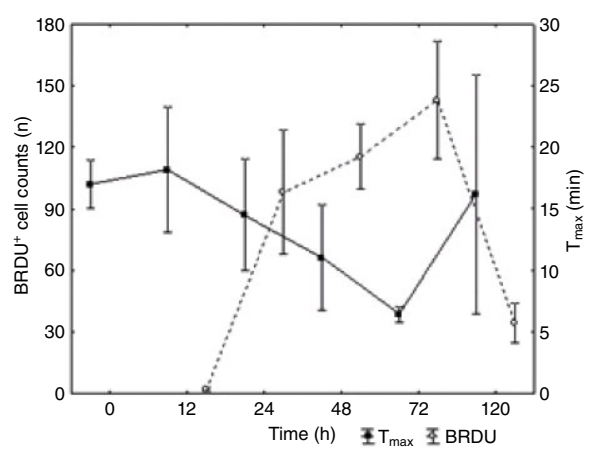

(b)

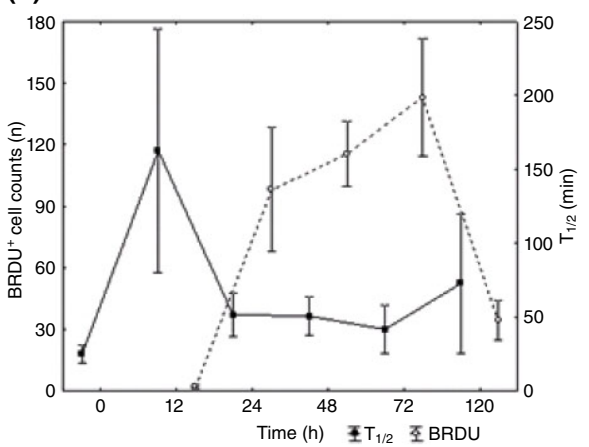

(d)

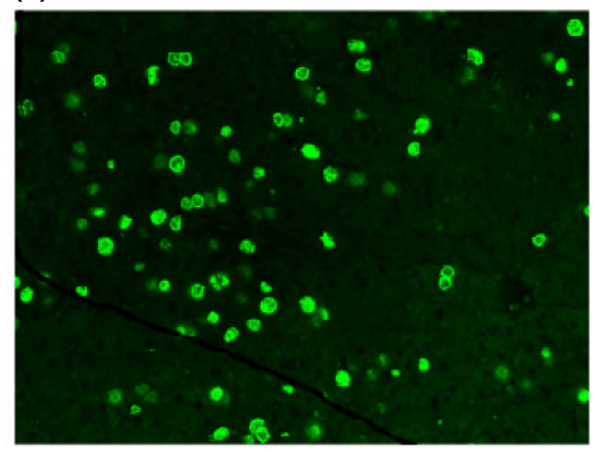

Fig. 2. Correlation between post-operative hepatic extraction fraction (HEF), T1/2, Tmax calculation after 99mTc-mebofrenin administration and bromodeoxyuridine deoxyribonucleic acid (BrdU) incorporation after rat $85 \%$ hepatectomy. (a) Positive correlation between hepatocyte proliferation evaluated by BrdU staining and HEF after rat $85 \%$ hepatectomy. (b) Negative correlation between hepatocyte proliferation evaluated by BrdU staining and T1/2 after rat $85 \%$ partial hepatectomy. (c) Negative correlation between hepatocyte proliferation evaluated by BrdU staining and Tmax after rat $85 \%$ partial hepatectomy. (d) Hepatocyte proliferation evaluated by BrdU staining $24 \mathrm{~h}$ after rat $85 \%$ hepatectomy.

results of scintigraphic study with $99 \mathrm{mTc}-$ mebrofenin and the evaluation of hepatocellular proliferation by immunohistochemistry using monoclonal anti-Ki67, which is a marker of cell proliferation. ${ }^{22}$ Moreover, the authors also found that the $99 \mathrm{mTc}$-mebrofenin uptake was the most sensitive of all functional methods to evaluate hepatic function during liver regeneration in standardized rat model. ${ }^{21}$

However, despite attempts, the obtained experimental data have not been much applied or used in clinical practice (new treatment strategies), and it was this motive that led us to conduct this clinical research. The two questions we addressed in this study are the following: First, do the same features of liver regenerations observed in animals apply to the regeneration of the human livers? Second, can our results be applied in our daily clinical practice?

Concerning the above, our study demonstrated a surprising capacity of human liver cells to regenerate, resulting in an early liver regeneration. This regeneration occurs early enough to normalize hepatic function (within 5 days after minor or major liver resection). Nevertheless, hepatic depuration needs more time in the earlier post-operative period (T5), which improves later reaching almost normal values at T30. The delay on T1/2 can be explained by the non-anatomic liver regeneration associated with the latter cholangiocyte regeneration compared with the hepatocyte regeneration verified in animal models of liver regeneration. ${ }^{5}$ In fact, the latter cholangiocyte regeneration can be the cause of the longer T1/2, despite the fact that the hepatocyte regeneration is already done. Liver regeneration is difficult to study in humans, and despite extensive research on the mechanisms responsible for the complex recovery of hepatocellular volume and function that occur during liver regeneration, they are not completely understood.

CT volumetry is considered the gold standard method to evaluate liver volumetry. However, performing a CT volumetry study $24 \mathrm{~h}$ or $48 \mathrm{~h}$ after surgery is not easy considering patient's condition after complex liver surgery. Volumetric tests (e.g. CT volumetry), however, has some limitations because it evaluates the volume but does not distinguish functional from non-functional hepatic 'volume'. Moreover, CT liver volume is an indirect measurement of hepatic function because the method assumes a homogeneous distribution function, a reality that is not valid particularly in patients with cirrhosis. ${ }^{20-22}$ Additionally, there is a poor correlation between the volumetric regeneration and functional regeneration, particularly during the first days after hepatectomy, and in our study it was essential to use functional evaluation tests to assess the recovery process of liver. $^{20,22}$

The most frequently used test for evaluating preoperative liver function is the indocyanine green (ICG) clearance test, which has been shown to be a better indicator of liver function than the ChildPugh classification. ICG is a tricarbocyanine dye, exclusively removed by the hepatocytes and excreted into the bile. Alternatively, $99 \mathrm{mTc}$ labelled iminodiacetic acid analogues, transported in blood 
(a)
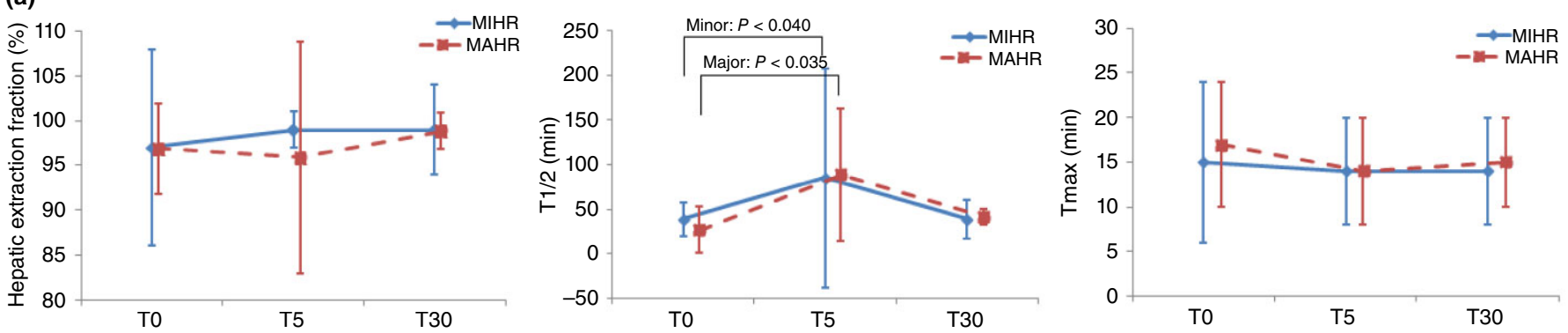

(b)

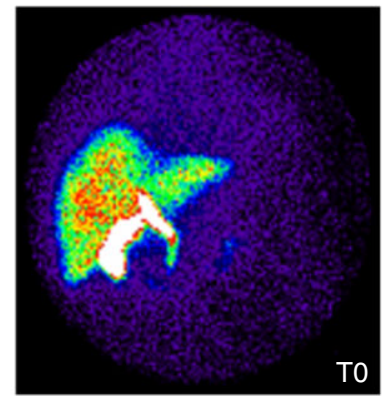

$\mathrm{HEF}=100 \% ;$ Tmax = $12 \mathrm{~min}$; $\mathrm{T} 1 / 2=39.6 \mathrm{~min}$

(c)

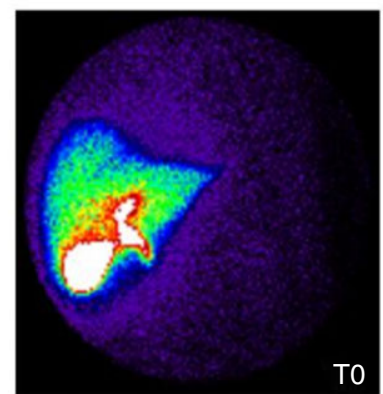

$\mathrm{HEF}=100 \% ;$ Tmax $=11 \mathrm{~min}$; $\mathrm{T} 1 / 2=39.6 \mathrm{~min}$

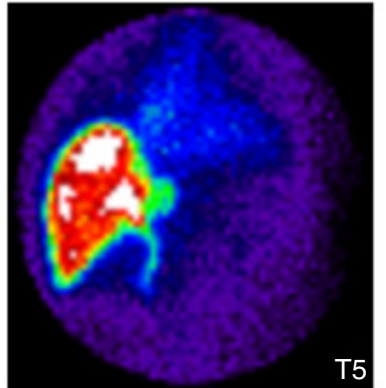

$\mathrm{HEF}=100 \% ; \mathrm{Tmax}=10 \mathrm{~min}$; $\mathrm{T} 1 / 2=47 \mathrm{~min}$

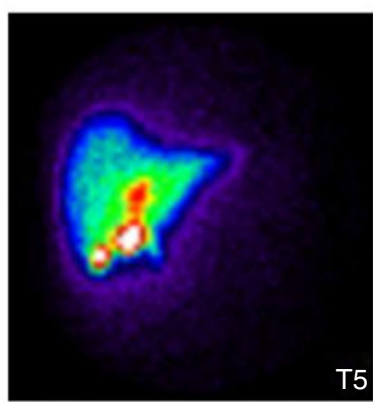

$\mathrm{HEF}=100 \%$; $\mathrm{Tmax}=8 \mathrm{~min}$; $\mathrm{T} 1 / 2=26 \mathrm{~min}$

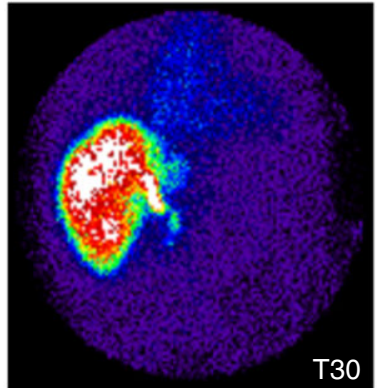

$\mathrm{HEF}=100 \%$; Tmax = $12 \mathrm{~min}$; $\mathrm{T} 1 / 2=38.7 \mathrm{~min}$

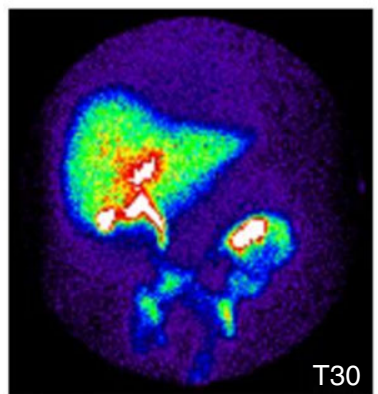

$\mathrm{HEF}=100 \% ;$ Tmax $=9 \mathrm{~min}$; $\mathrm{T} 1 / 2=30 \mathrm{~min}$

Fig. 3. Study of dynamic human liver regeneration. (a) Hepatic extraction fraction (HEF), Tmax and T1/2 in each one of the evaluations (T0, T5 or T30 days after hepatectomy) as showed graphically. Time made no differences on HEF and Tmax; however, it had a significant statistical effect on T1/2 ( $P$ value $<0.001$ ), which is visible graphically, in individuals submitted to minor hepatic resection (MIHR) $(P<0.040)$, as well as those who underwent major hepatic resection (MAHR) $(P<0.035)$. (b) $99 \mathrm{mT}$ c-mebrofenin scintigraphic images obtained on a patient with colorectal liver metastases submitted to a major hepatic resection (MAHR) 1 day before hepatectomy (T0), 5 days (T5) and 30 days after hepatectomy (T30). (c) 99mTc-mebrofenin scintigraphic images obtained on a patient with colorectal liver metastases submitted to a minor hepatic resection (MIHR) 1 day before hepatectomy (T0), 5 days (T5) and 30 days after hepatectomy (T30).

by binding to albumin in the same manner as ICG, can be used for hepatobiliary scintigraphy in the assessment of liver function. Both ICG and $99 \mathrm{mTc}-\mathrm{meb}$ rofenin are excreted in bile by the hepatocytes by the ATP-dependent export pump multidrug resistance-associated protein 2, without undergoing biotransformation during their transit through the hepatocyte. Therefore, these agents are well suited for the study of hepatic transport. In fact, $99 \mathrm{mTc}$-mebrofenin uptake rate assessed by scintigraphy is a valid method for determining liver function, and correlates well with ICG clearance. ${ }^{23}$ On the other hand, a correlation between liver function evaluated by ICG clearance and hepatic parenchymal cell volume was also demonstrated. ${ }^{24}$
During the regeneration process of the liver, a strong correlation between conventional liver volume and liver functional volume was demonstrated. ${ }^{1-9,20}$ As liver regeneration is associated with liver volume and/or liver weight increase, what we observed with the proposed study is that hepatocellular function recovery occurs before the liver regeneration process. Our results coming from animal studies by analysing DNA synthesis using BrdU incorporation and the liver/bodyweight ratio also support the same conclusion. Additionally, these results correlate with those observed in human patients, showing that the human liver regeneration is fast, nonanatomic and certainly complex. 
How could the new knowledge be applied in clinical practice?

The surprising replicative capacity of human hepatocytes has a very important clinical significance as it has allowed us to address the second question - the clinical applicability in real life of such observation. To begin with, the implications of the observed results can greatly influence the ongoing oncological practices of adjuvant chemotherapy, possibly resulting in treatments administered much earlier after surgical resection than the 3-week interval currently applied by most oncologists. Similarly, the same applies to liver resection following portal embolization. With a regeneration taking place in the first 5 days, the planned liver resection can be performed much earlier also. Furthermore, several conditions can influence liver regeneration, including the extent of resection, diabetes and the presence of underlying liver disease..$^{25,26}$ In case of liver transplantation, the duration of the cold ischemic period and the presence of organ rejection are additional risk factors. The HEF calculated with 99mTc-mebrofenin can give a reliable assessment of liver function during regeneration, and is therefore important after transplantation or partial hepatectomy.

\section{Conclusions}

During the last few years, there have been major advances in understanding the mechanisms of liver regeneration. These advances derive, to a great extent, from the studies of liver regeneration conducted in animal models, as the results shown by us concerning the positive correlation between HEF kinetics and LW/AW ratio after hepatectomy, or the positive correlation between hepatocyte proliferation evaluated by BrdU staining and HEF after partial hepatectomy.

In parallel with the experimental work, human liver transplantation, such as cadaveric split grafts and living donors, continues to increase, stimulating hepatologists and surgeons to learn more about the mechanisms that regulate and promote regeneration. Our data based on animal and clinical results support the view that human liver regeneration is early, fast, non-anatomical and functionally complete 5 days after hepatectomy. The findings have an important clinical impact as they can lead to a strategy change of the oncological therapeutic approaches like the early start of adjuvant chemotherapy after hepatectomy.

\section{References}

1. Fausto N, Kimberly JR. Mechanisms of liver regeneration and their clinical implications. J. Hepatobiliary Pancreat. Surg. 2005; 2: 181-9.

2. Alison MR. Regulation of hepatic growth. Physiol. Rev. 1986; 66: 499541.

3. Fausto N, Webber EM. Control of liver growth. Crit. Rev. Eukaryot. Gene Expr. 1993; 3: 117-35.

4. Michapoulos GK, Defrances MC. Liver regeneration. Science 1997; 276: $60-6$.

5. Fausto N. Liver regeneration. J. Hepatol. 2000; 32 (Suppl. 1): 19-31.

6. Fausto N, Campbell JS, Rihele KJ. Liver regeneration. Hepatology 2006; 43: s45-s53.
7. Michapoulos GK. Liver regeneration. J. Cell. Physiol. 2007; 213: 286300.

8. Oertel M, Shafritz DA. Stem cells, cell transplantation and liver repopulation. Biochim. Biophys. Acta 2008; 1782: 61-74.

9. Cantré D, Schuette H, Hildebrandt A et al. Nitric oxide reduces organ injury and enhances regeneration of reduced-size livers by increasing hepatic arterial flow. Br. J. Surg. 2008; 95: 785-92.

10. Francavilla A, Ove P, Polimeno L et al. Regulation of liver size and regeneration: importance in liver transplantation. Transplant. Proc. 1988; 20: 494-7.

11. Kawasaki S, Makuuchi M, Ishizone $\mathrm{S}$ et al. Liver regeneration in recipients and donors after transplantation. Lancet 1992; 339: 580-1.

12. Tralhao JG, Roudier J, Morosan S et al. Paracrine in vivo inhibitory effects of hepatitis B virus X protein (HBx) on liver cell proliferation: an alternative mechanism of HBx-related pathogenesis. Proc. Natl. Acad. Sci. U. S. A. 2002; 99: 6991-6.

13. Palmes D, Spiegel H. Animal models of liver regeneration. Biomaterials 2004; 25: 1601-11.

14. Tralhao JG, Botelho MF, Abrantes AM et al. Interest of the study of the hepatic extraction fraction by radioisotopic methods in patients with hepatic tumors undergoing hepatectomy [abstract]. Eur. J. Nucl. Med. Mol. Imaging 2006; 33 (Suppl. 2): S201.

15. Lima JMP, Lima JJP, Isidoro $\mathrm{J}$ et al. A new method for quantification of hepatobiliary scintigraphy using Tc-99m mebrofenin. A comparative study. Rev. Esp. Med. Nucl. 2003; 22: 244-9.

16. Tralhão JG, Abrantes AM, Hoti E et al. Hepatic extraction fraction: interest of its quantification in patients with hepatic tumours. Hepatogastroenterology 2012; 59: 1507-11.

17. Pugh RN, Murray-Lyon IM, Dawson JL et al. Transection of oesophagus for bleeding oesophageal varices. Br. J. Surg. 1973; 60: 646-9.

18. International Commission of Radiological Protection. Pamphlet No. 53 by the ICRP (International Commission of Radiological Protection), 1988.

19. de Graaf W, Veteläinen RL, de Bruin K et al. 99mTc-GSA scintigraphy with SPECT for assessment of hepatic function and functional volume during liver regeneration in a rat model of partial hepatectomy. J. Nucl. Med. 2008; 49: 122-8.

20. de Graaf W, Bennink RJ, Heger M, Maas A et al. Quantitative assessment of hepatic function during liver regeneration in a standardized rat model. J. Nucl. Med. 2011; 52: 294-302.

21. Bennink RJ, Dinant S, Erdogan D et al. Preoperative assessment of postoperative remnant liver function using hepatobiliary scintigraphy. $J$. Nucl. Med. 2004; 45: 965-71.

22. Erdogan D, Heijnen BH, Bennink RJ et al. Preoperative assessment of liver function: a comparison of 99m TC-Mebrofenin scintigraphy with indocyanine green clearance test. Liver Int. 2004; 24: 117-23.

23. Hashimoto M, Watanabe G. Hepatic parenchymal cell volume and the Indocyanine Green tolerance test. J. Surg. Res. 2000; 92: 222-7.

24. Veteläinen R, van Vliet AK, van Gulik TM. Severe steatosis increases hepatocellular injury and impairs liver regeneration in a rat model of partial hepatectomy. Ann. Surg. 2007; 245: 44-50.

25. Yokoyama Y, Nagino M, Nimura Y. Mechanisms of hepatic regeneration following portal vein embolization and partial hepatectomy: a review. World J. Surg. 2007; 31: 367-74.

26. Mazzaferro V, Regalia E, Doci R et al. Liver transplantation for the treatment of small hepatocellular carcinomas in patients with cirrhosis. N. Engl. J. Med. 1996; 334: 693-9. 